\title{
Chemical chaperone 4-phenylbutyric acid protects H9c2 cardiomyocytes from ischemia/reperfusion injury by attenuating endoplasmic reticulum stress-induced apoptosis
}

\author{
LIAN JIAN ${ }^{1}$, YUAN LU ${ }^{1}$, SHAN LU ${ }^{2}$ and CHENGZHI LU ${ }^{1}$ \\ ${ }^{1}$ Cardiovascular Department, Tianjin First Central Hospital, Tianjin 300192; ${ }^{2}$ Radiology Department, \\ Tianjin Medical University Metabolic Diseases Hospital, Tianjin 300000, P.R. China
}

Received April 5, 2015; Accepted February 2, 2016

DOI: $10.3892 / \mathrm{mmr} .2016 .5063$

\begin{abstract}
Myocardial ischemia/reperfusion (I/R) is a potential contributor to high rates of mortality in several cardiovascular diseases. I/R initiates the unfolded protein response and endoplasmic reticulum (ER) stress, which may lead to apoptotic pathways and exaggerate I/R injury. 4-phenylbutyric acid (4-PBA), a low molecular weight, terminal aromatic substituted fatty acid, has been reported to function as an ER chaperone. The aim of the present study was to investigate whether 4-PBA is able to reduce ER stress-induced apoptosis and prevent cardiomyocyte damage during the process of $\mathrm{I} / \mathrm{R}$ in vitro. Accordingly, the rat cardiomyocyte line, $\mathrm{H} 9 \mathrm{c} 2$, was treated with hypoxia/reoxygenation as an I/R model in vitro. Myocardium apoptosis was determined with TUNEL staining. The expression of ER stress-related proteins were examined by western blotting. The resulting data showed that I/R activates the ER stress proteins, glucose-regulated protein 78 , activating transcription factor 6 and protein kinase RNA-like endoplasmic reticulum kinase, which were all reduced by pretreatment with 4-PBA. In addition, pretreatment with 4-PBA significantly inhibited the expression levels of pro-apoptotic proteins, C/EBP homologous protein, $\mathrm{B}$ cell lymphoma (Bcl-2)-associated $\mathrm{X}$ protein and phosphorylated c-Jun $\mathrm{N}$-terminal kinase, and enhanced the expression of the anti-apoptotic protein $\mathrm{Bcl}-2(\mathrm{n}=3 ; \mathrm{P}<0.05)$. The data demonstrated that I/R initiates ER stress-associated apoptotic pathways, and 4-PBA pretreatment protected the cardiomyocytes from I/R-induced cell death. To the best of our knowledge, the present study is the first to report on the cell repair mechanism of 4-PBA against I/R damage in cardiomyocytes based on ER stress-associated apoptotic pathways.
\end{abstract}

Correspondence to: Dr Chengzhi Lu, Cardiovascular Department, Tianjin First Central Hospital, 24 Fukang Road, Nankai, Tianjin 300192, P.R. China

E-mail: luchengzhi2015@163.com

Key words: ischemia/reperfusion, 4-phenylbutyric acid, unfolded protein response, endoplasmic reticulum stress, apoptosis

\section{Introduction}

Cardiovascular diseases are the leading contributor to mortality rates worldwide. According to the World Health Organization (WHO), by $2030,>23,000,000$ individuals will succumb to mortality from cardiovascular diseases annually. Myocardial ischemia is one of the main consequences of cardiovascular diseases and can be characterized into several forms (1). Although timely reperfusion is essential for recovery of the ischemia myocardium, sudden restoration of blood flow may exaggerate myocardial injury (2,3). A study in 1960 reported that the reperfusion of ischemic myocardium may aggravate myocardial injury in dogs, which is known as myocardial ischemia/reperfusion (I/R) injury $(4,5)$. Although the underlying mechanism regulating myocardial injury induced by $\mathrm{I} / \mathrm{R}$ remains to be fully elucidated, previous evidence suggests that apoptosis is an essential form of cell death in I/R injury (6). Myocardial apoptosis is initiated shortly following ischemia, however, for substantial apoptotic cell death to occur, reperfusion is necessary.

The endoplasmic reticulum (ER) is an organelle with an essential role in multiple cellular processes, including sensing oxidative stress, maintaining calcium homeostasis and triggering apoptotic signaling $(7,8)$. I/R injury impairs key functions of $\mathrm{ER}$, including the synthesis, folding and sorting of proteins, and induces a stress state, which activates the evolutionarily conserved unfolded protein response (UPR) (8). ER stress is initially sensed by three ER transmembrane receptors, protein kinase RNA-like endoplasmic reticulum kinase (PERK), activating transcription factor 6 (ATF6) and inositol-requiring enzyme 1 (IRE1), which monitor the homeostasis of the ER and trigger the UPR (9). The UPR rapidly attenuates general protein synthesis, induces the expression of ER chaperone proteins and enhances the degradation of misfolded/unfolded proteins. Although the UPR is primarily an adaptive response, if the stress persists, the ER stress receptors can also trigger pro-apoptotic pathways to initiate cell death (10). Sustained ER stress, acting through PERK, ATF6, and IRE1, induces expression of the pro-apoptotic protein $\mathrm{C} / \mathrm{EBP}$ homologous protein (CHOP). The overexpression of CHOP induces apoptosis through a B cell lymphoma-2 (Bcl-2)-inhibiting mechanism and c-Jun amino-terminal kinase (JNK) enhancing pathways $(10,11)$. Sufficient evidence indicates 
that high protein levels of CHOP are associated with cell death by ER stress-independent mechanisms. Inhibiting the apoptotic process induced by the ER stress pathway may prevent the loss of contractile cells, minimize cardiac injury induced by I/R, and slow the occurrence of myocardial stunning and heart failure (12).

4-phenylbutyric acid (4-PBA) is a low molecular weight, terminal aromatic substituted fatty acid and a non-toxic pharmacological compound, which is currently approved for clinical use as an ammonia scavenger in disorders of the urea cycle in children (13). This molecule is also used for the treatment of sickle cell disease due to its capacity to activate $\beta$-globin transcription (14). Previously, 4-PBA was found acting as a chemical chaperone in the ER, improving ER folding capacity and facilitating the trafficking of mutant proteins. The underlying mechanism predominantly relies on its physicochemical properties, which allow the stabilization of peptide structures, improving the luminal folding capacity and the traffic of aberrant proteins (15-17). It has been reported that 4-PBA shows protective effects against ER stress-induced neuronal and myocardial cell death (18). Thus, the use of 4-PBA may provide a therapeutic approach for inhibiting the pathologic process of ER stress and cell death induced by I/R.

However, the mechanism underlying the activation of ER stress, as well as the function of 4-PBA in the injured myocardium, remain to be elucidated. Understanding how 4-PBA responds to ER stress is necessary to assess its contribution to cardioprotection. The present study aimed to investigate the effects of the chemical chaperone, 4-PBA, on ER stress and examine the mechanisms underlying these effects in a model of myocardial cell death induced by I/R injury.

\section{Materials and methods}

Reagents. The following reagents were purchased from Sigma-Aldrich (St. Louis, MO, USA): 4-PBA, 3-(4,5-dimethylthiazol-2-yl)-2,5-diphenyl tetrazolium bromide (MTT), 5,5',6,6'-tetrachloro-1,1',3,3'-tetraethyl benzimidazolyl carbocyanine iodide (JC-1) and dimethyl sulfoxide (DMSO). Dulbecco's modified Eagle's medium (DMEM), fetal bovine serum (FBS), and phosphate-buffered saline (PBS) were purchased from Gibco; Thermo Fisher Scientific, Inc. (Waltham, MA, USA). A terminal deoxynucleotidyl transferase-mediated biotinylated UTP nick end-labeling (TUNEL) kit was purchased from Invitrogen; Thermo Fisher Scientific, Inc. Primary antibodies against glucose-regulated protein 78 (Grp78; sc-13968), ATF6 (sc-22799), PERK (sc-13071), CHOP (sc-7351), JNK (sc-7345), phosphorylated (P)-JNK (sc-6254), BAX (sc-493), Bcl-2 (sc-492), and $\beta$-actin (sc-47778) were obtained from Santa Cruz Biotechnology, Inc. (Santa Cruz, CA, USA). Secondary antibodies were purchased from Beijing ComWin Biotech Co., Ltd. (Beijing, China).

Cell line and cell culture. Cells of the rat $\mathrm{H} 9 \mathrm{c} 2$ cardiomyocyte cell line were cultured in DMEM with 10\% FBS and 1\% antibiotic-antimycotic (penicillin and streptomycin; Gibco; Thermo Fisher Scientific, Inc.). When cell confluency reached to $70-80 \%$, the $\mathrm{H} 9 \mathrm{c} 2$ cells, maintained at $37^{\circ} \mathrm{C}$ with $5 \% \mathrm{CO}_{2}$, were used for experiments. For all the treatment groups, the $\mathrm{H} 9 \mathrm{c} 2$ cells $(100,000$ cells/ml) were pre-incubated with or without different concentrations $(0.5,1,2.5$ and $5 \mathrm{mM})$ of 4-PBA, dissolved in $\mathrm{PBS}$, for $24 \mathrm{~h}$ prior to initiating I/R. A simulated I/R cell model was used, as described previously (19) with modifications. The H9c2 cells were exposed to ischemia by replacing the DMEM with ischemic buffer (DMEM without D-glucose; pH 6.3; Gibco; Thermo Fisher Scientific, Inc.). Subsequently, the cells were incubated in a Modular Incubator Chamber (Billups-Rothenberg, Del Mar, CA, USA) in an atmosphere of $95 \%$ nitrogen and $5 \% \mathrm{CO}_{2}$ at $37^{\circ} \mathrm{C}$ for $6 \mathrm{~h}$. Following ischemia, reperfusion was initiated by incubating the cells in DMEM at $37^{\circ} \mathrm{C}$ with $5 \% \mathrm{CO}_{2}$ for different durations. In the normoxic control groups, the cells were incubated with DMEM in an atmosphere of $5 \% \mathrm{CO}_{2}$ at $37^{\circ} \mathrm{C}$.

Cell viability analysis. Cell viability was determined using an MTT assay and cell death was measured using a lactate dehydrogenase (LDH) assay, as previously reported (20). Briefly, the H9c2 cardiomyocytes were seeded at a density of $5 \times 10^{3}$ cells per well into 96 -well plates and, at the end of the respective treatments, the cells were incubated with MTT solution $(1 \mathrm{mg} / \mathrm{ml}$ final concentration stock solution in PBS per well) at $37^{\circ} \mathrm{C}$ for $4 \mathrm{~h}$. The medium was then removed, and the formazan crystals were dissolved with $150 \mu \mathrm{l}$ DMSO. The absorbance at $570 \mathrm{~nm}$ was determined using a microplate reader (Spectra Fluo, Tecan, Sunrise, Austria). The experiments were repeated in triplicate and data were expressed as the percentages of the control. The LDH assay was used to evaluate myocardial cell death. Following I/R treatment with or without 4-PBA incubation, the medium of the cardiomyocytes was collected to measure LDH release using LDH assay kits, according to the manufacturer's protocol.

Determination of cardiomyocyte apoptosis. Myocardial apoptosis was assessed using TUNEL staining, according to the manufacturer's protocol. The apoptotic index was expressed as the number of apoptotic cells of all cardiomyocytes per field, following the assessment of 20 randomly-selected fields per specimen. Positive (DNase-treated) and negative (no addition of terminal transferase) control tissue sections were incorporated into each assay. Individual nuclei were visualized at 200x magnification, and all measurements were performed in a blinded-manner.

Measurement of mitochondrial membrane potential $(\Psi m)$. The changes in $\Psi \mathrm{m}$ were detected using JC-1 staining. The $\mathrm{H} 9 \mathrm{c} 2$ cardiomyocytes were cultured $\left(1 \times 10^{5}\right.$ cells/well) in poly-L-lysine-coated six-well plates. Following the pretreatment, the cells were incubated with $\mathrm{JC}-1$ ( $2 \mu \mathrm{M}$ final concentration) at $37^{\circ} \mathrm{C}$ in the dark for $15 \mathrm{~min}$. The cells were then washed three times with PBS and examined under a Nikon TE300 confocal fluorescence microscope equipped with an argon laser (Nikon Coproration, Tokyo, Japan).

Western blotting. For immunoblotting, the cells were lysed with RIPA buffer, containing 50 mM Tris-HCl (pH 7.5), $100 \mathrm{mM}$ $\mathrm{NaCl}$ and $1 \%$ Triton $\mathrm{X}-100$, with protease inhibitors (aprotinin, leupeptin, phenylmethylsulfonyl fluoride and pepstatin) and phosphatase inhibitor (Sigma cocktail; Sigma-Aldrich). Following the lysate centrifugation for $15 \mathrm{~min}$ at $12,000 \mathrm{x} \mathrm{g}$ at $4^{\circ} \mathrm{C}$, the supernatants were collected by removing insoluble 
materials. Protein was quantified using a BCA Protein Assay Kit (Beijing ComWin Biotech Co, Ltd.). For Western blot analysis, an equal quantity of protein $(50-100 \mu \mathrm{g})$ was loaded in each well and separated by $10-15 \%$ sodium dodecyl sulfate-polyacrylamide gel electrophoresis. The separated proteins were then transferred from the gel onto polyvinylidinene fluoride membranes (EMD Millipore, Bedford, MA, USA) and blocked in 5\% non-fat dry milk prepared in $1 \mathrm{X}$ Tris-buffered saline with 20\% Tween-20 (TBST; Beijing ComWin Biotech Co., Ltd.) for $2 \mathrm{~h}$. The membranes were incubated overnight at $4^{\circ} \mathrm{C}$ with the following primary antibodies: Polyclonal rabbit anti-Grp78 (1:500), monoclonal mouse anti-CHOP (1:200), polyclonal rabbit anti-BAX (1:200), polyclonal rabbit anti-Bcl-2 (1:200), polyclonal rabbit anti-PERK (1:500), polyclonal rabbit anti-ATF6 (1:500), monoclonal mouse anti-P-JNK (1:200), monoclonal mouse anti-JNK (1:200) and monoclonal mouse anti- $\beta$-actin $(1: 1,000)$. Following washing of the membranes three times with $1 \mathrm{X}$ TBST, the membranes were probed with the following secondary antibodies $(1: 1,000)$ for $2 \mathrm{~h}$ at room temperature: Horseradish peroxidase (HRP)-conjugated goat anti-rabbit $\operatorname{IgG}(\mathrm{CW} 0103 \mathrm{M})$ and HRP-conjugated goat anti-mouse IgG (CW0102M). Following $30 \mathrm{~min}$ of washing with TBST, the membranes were visualized using an ECL Plus detection system (Beyotime Institute of Biotechnology, Haimen, China) and the relative band densities were measured using a Fluor Chem FC2 system (NatureGene Corp., Beijing, China).

Statistical analysis. The mean values were calculated from the data obtained from three investigations in each group and at least three separate in vitro experiments. GraphPad Prism version 5.0 (GraphPad Software, Inc., La Jolla, CA, USA) was used to perform all statistical analyses. The results were compared using one-way analysis of variance and Tukey's post-hoc test to identify specific differences between groups. $\mathrm{P}<0.05$ was considered to indicate a statistically significant difference.

\section{Results}

$I / R$-induced cell death is dependent on the duration of reperfusion. In order to investigate whether 4-PBA was able to protect against cardiac injury induced by I/R in vitro, the present study first determined the effect of reperfusion duration on cell death. The H9c2 cardiomyocytes were exposed to hypoxia for $6 \mathrm{~h}$, as previously reported (19), followed by reoxygenation for different periods of time $(0.5,1,2,4,8$ and $12 \mathrm{~h})$ to mimic in vitro $\mathrm{I} / \mathrm{R}$ conditions. Cell viability was then detected using an MTT assay and cell death was evaluated using an LDH assay. As shown in Fig. 1A, the cells in control group were considered $100 \%$ viable. Hypoxia and reoxygenation caused a decrease in cell viability in a time-dependent manner. The viability of the cardiomyocytes at $12 \mathrm{~h}$ post-reoxygenation was $\sim 57 \%$. In addition, LDH leakage was measured as a biomarker of cell death. As shown in Fig. 1B, reoxygenation induced the release of LDH in a time-dependent manner, compared with the untreated control group. LDH leakage increased rapidly between 0.5 and $8 \mathrm{~h}$ post-reoxygenation and reached a peak at $12 \mathrm{~h}$, which was $\sim 8$-fold of that in the untreated control group. Based on these results, ischemia for $6 \mathrm{~h}$ and reperfusion for $12 \mathrm{~h}$ were selected for the in vitro $\mathrm{I} / \mathrm{R}$ conditions in the following experiments.
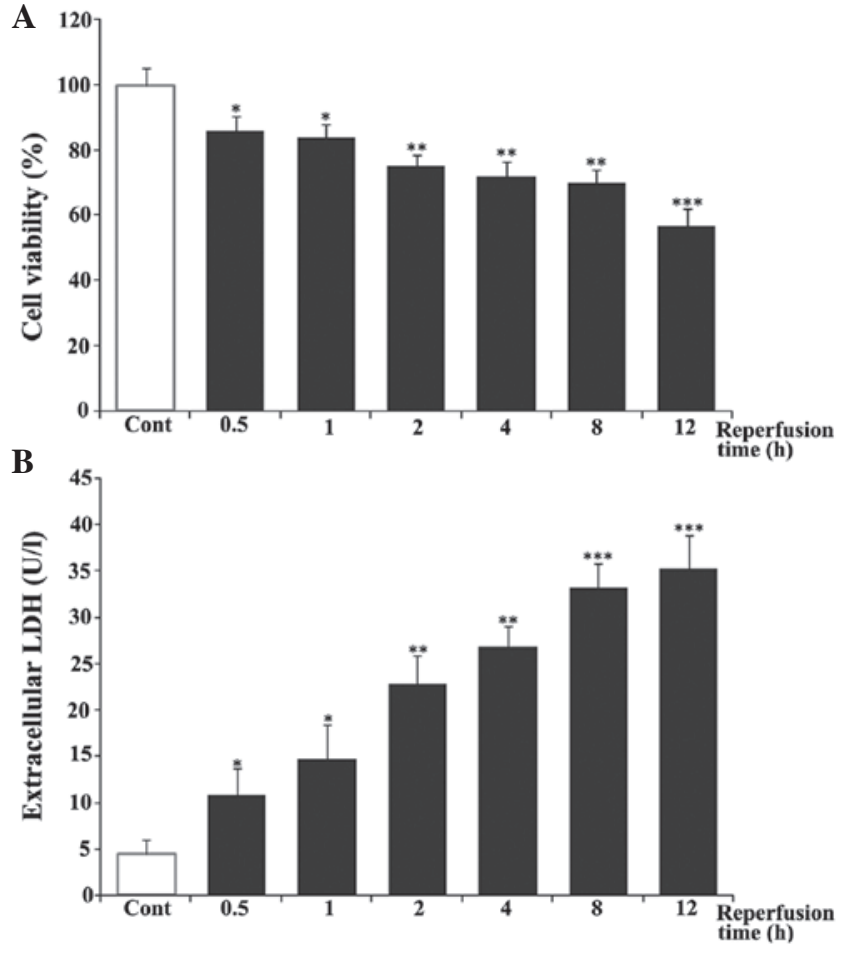

Figure 1. Effects of reperfusion time on cell viability and LDH release in $\mathrm{H} 9 \mathrm{c} 2$ cardiomyocytes. H9c2 cardiomyocytes were exposed to $6 \mathrm{~h}$ of hypoxia, followed by reoxygenation for $0.5,1,2,4,8$ or $12 \mathrm{~h}$. (A) Cell viability was then examined using a 3-(4,5-dimethylthiazol-2-yl)-2,5-diphenyl tetrazolium bromide assay. (B) Cell death was measured using an LDH assay kit. The results are represented as the mean \pm standard deviation from three independent experiments. ${ }^{*} \mathrm{P}<0.05,{ }^{* *} \mathrm{P}<0.01$ and ${ }^{* * *} \mathrm{P}<0.001$, vs. Cont. Cont, untreated control; LDH, lactate dehydrogenase.

Treatment with 4-PBA prevented I/R injury-mediated myocardial cell death. To evaluate the effect of 4-PBA (Fig. 2A) on I/R injury on the rat $\mathrm{H} 9 \mathrm{c} 2$ cells, the cardiomyocytes were incubated with 4-PBA at a range of concentrations between 0 and $5 \mathrm{mM}$ for $24 \mathrm{~h}$, prior to mimicking I/R injury in vitro. MTT and $\mathrm{LDH}$ assays were performed to determine the role of 4-PBA in the prevention of I/R-induced damage. The data demonstrated that cell viability was significantly improved following treatment with 4-PBA at concentrations between 0.5 and $5 \mathrm{mM}$, in a dose-dependent manner, in triplicate experiments (Fig. 2B). No statistically significant differences were found in cell viability or cell death between the cells incubated with $5 \mathrm{mM}$ 4-PBA for $24 \mathrm{~h}$ and the control group (Fig. 2B). The level of LDH leakage in the I/R group was significantly higher, compared with that in the control group $(\mathrm{P}<0.001)$, whereas 4-PBA pretreatment decreased the levels of LDH (Fig. 2C). The results confirmed that 4-PBA pretreatment had the ability to inhibit the H9c2 cell death induced by $\mathrm{I} / \mathrm{R}$, and pretreatment of the cells with 4-PBA at $5 \mathrm{mM}$ for $24 \mathrm{~h}$ was used in the subsequent experiments.

Treatment with 4-PBA prevented the cell apoptosis induced by myocardial I/R injury. To examine whether 4-PBA has any effect on I/R-induced apoptosis in H9c2 cells, the present study performed aTUNEL assay and JC-1 staining. The nucleiof normal cells were stained by propidium iodide and those of apoptotic cells were stained green. The number of TUNEL-positive cells was manually counted in five randomly-selected fields, with the apoptotic index expressed as a percentage of the total counted cells (Fig. 3A). TUNEL staining showed that I/R increased the 
A<smiles>O=C(O)CCCc1ccccc1</smiles>

B

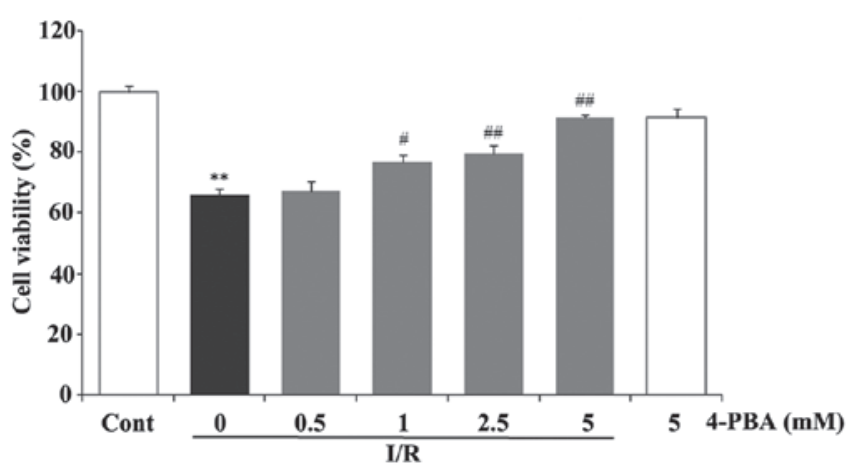

C

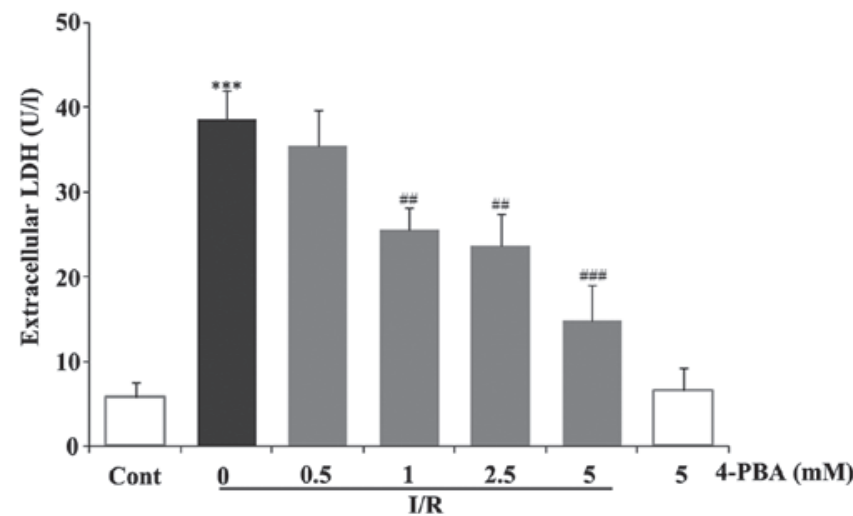

Figure 2. Effects of 4-PBA on cell viability and LDH release in I/R-induced H9c2 cells. H9c2 cardiomyocytes were incubated with different concentrations of 4-PBA $(0.5,1,2.5$ and $5 \mathrm{mM})$ for $2 \mathrm{~h}$ prior to exposure to hypoxia for $6 \mathrm{~h}$ and reoxygenation for $12 \mathrm{~h}$. (A) Chemical structure of 4-PBA. (B) Cell viability was determined using a 3-(4,5-dimethylthiazol-2-yl)-2,5-diphenyl tetrazolium bromide assay and expressed as a relative percentage of the control group. (C) LDH release was measured to determine cell death. The results are represented as the mean \pm standard deviation from three independent experiments. ${ }^{* *} \mathrm{P}<0.01$ and ${ }^{* * * *} \mathrm{P}<0.001$, vs. Cont; ${ }^{\#} \mathrm{P}<0.05,{ }^{\# \#} \mathrm{P}<0.01$ and ${ }^{\# \# \#} \mathrm{P}<0.001$, vs. I/R-treated cells. 4-PBA, 4-phenylbutyric acid; I/R, iscehemia/reperfusion; Cont, untreated control; $\mathrm{LDH}$, lactate dehydrogenase.

A
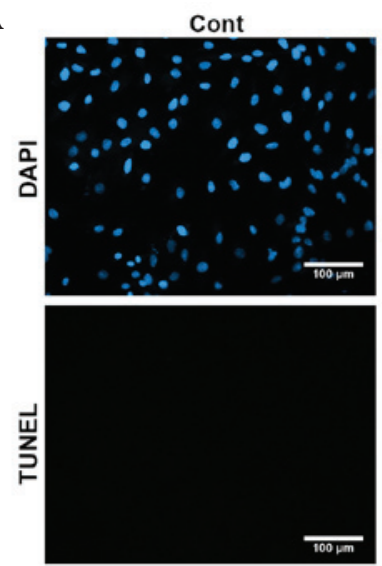

B

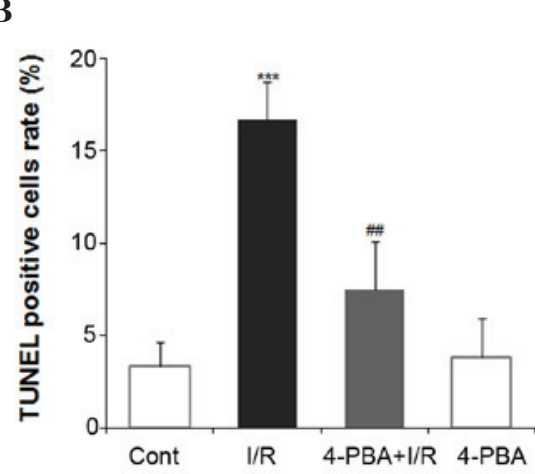

I/R
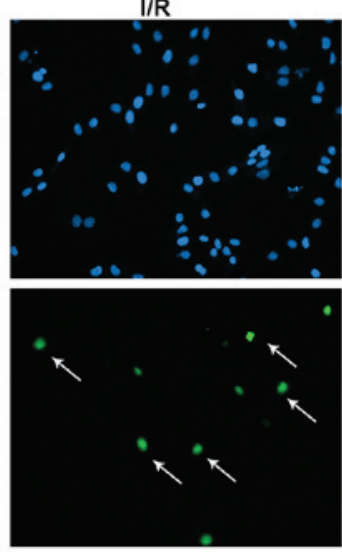

C
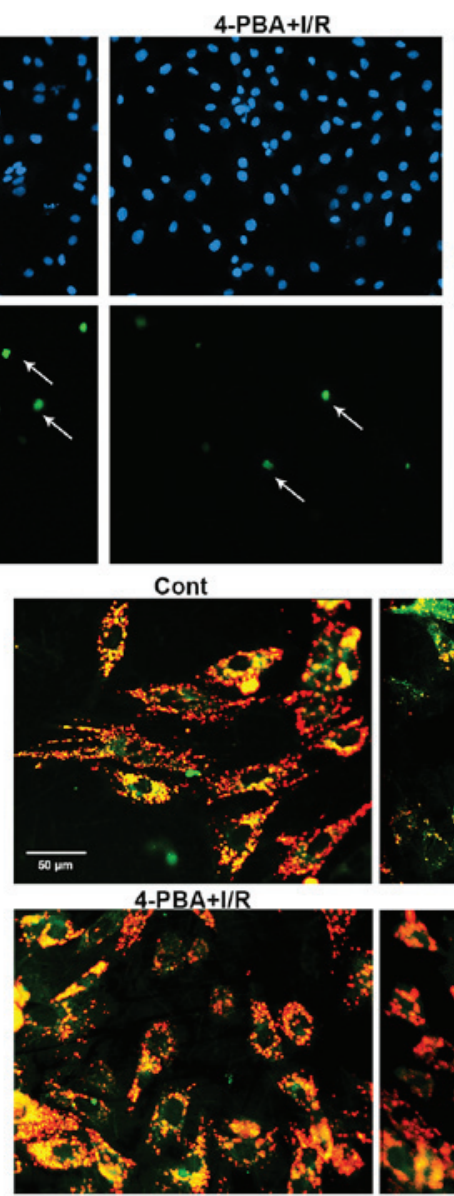
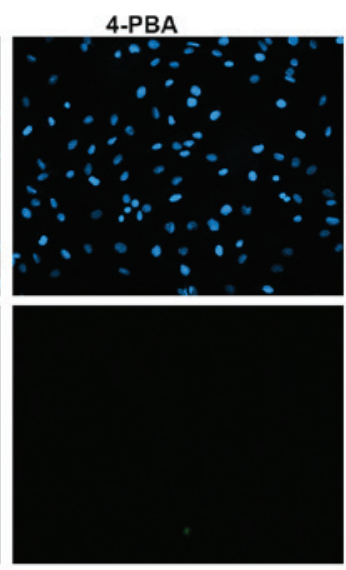

I/R
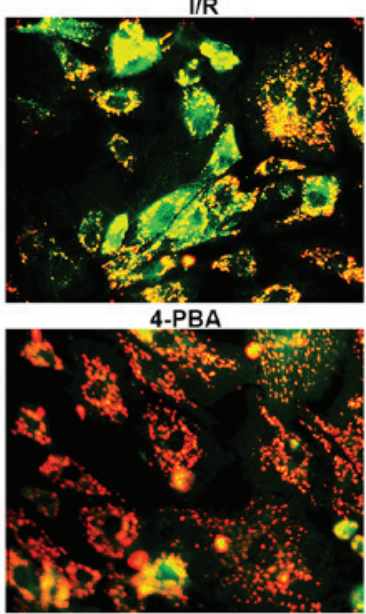

Figure 3. Protective effect of 4-PBA on I/R-induced apoptosis in H9c2 cells. The H9c2 cells were pre-incubated with 5 mM 4 PBA for 2 h, followed by 6 h of hypoxia and $12 \mathrm{~h}$ of reoxygenation. (A) Images of DNA fragmentation were detected using a TUNEL assay in the apoptotic H9c2 cells. Arrows represent TUNEL-positive cells. (B) Quantification of the TUNEL-positive cell rate. The results are expressed as the mean \pm standard deviation of three independent experiments. ${ }^{* * * *} \mathrm{P}<0.01$, vs. Cont; ${ }^{\# \#} \mathrm{P}<0.01$ vs. I/R. (C) H9c2 cardiomyocytes stained with JC-1 dye were visualized using fluorescence microscopy. 4-PBA, 4-phenylbutyric acid; I/R, iscehemia/reperfusion; Cont, untreated control; TUNEL, terminal deoxynucleotidyl transferase-mediated biotinylated UTP nick end-labeling. 

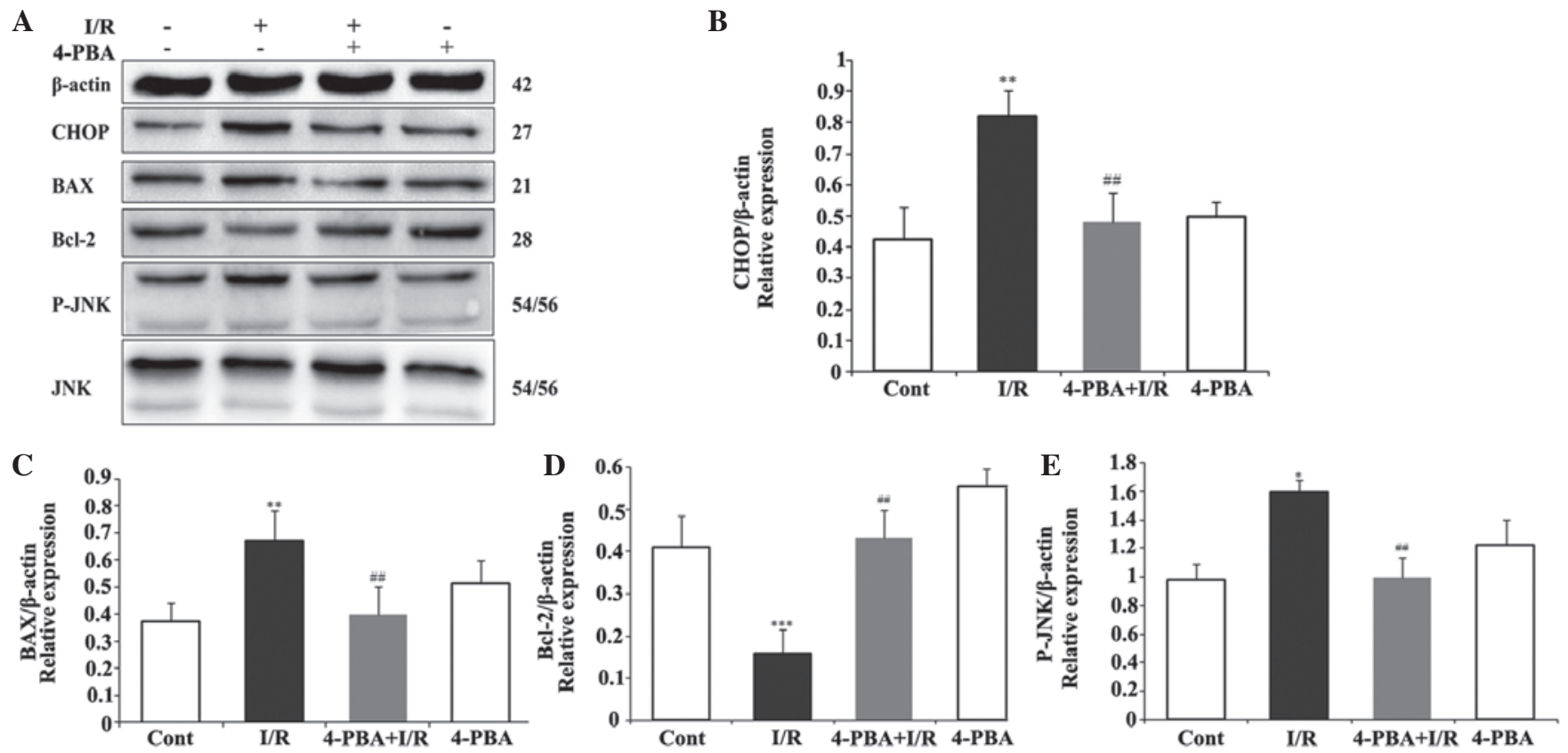

Figure 4. Effects of the expression of 4-PBA on apoptosis-associated proteins in I/R-induced H9c2cells. The H9c2 cells were exposed or not exposed to I/R, with or without $5 \mathrm{mM}$ 4-PBA incubation. (A) 4-PBA suppressed the I/R-induced upregulation of CHOP, BAX and P-JNK, and downregulation of Bcl-2. The normalized ratio of (B) CHOP, (C) BAX and (D) Bcl-2 to $\beta$-actin, and (E) P-JNK to JNK were determined by densitometry. The results are expressed as the mean \pm standard deviation of three independent experiments. ${ }^{*} \mathrm{P}<0.05,{ }^{* *} \mathrm{P}<0.01$ and ${ }^{* * * *} \mathrm{P}<0.001$ vs. Cont; ${ }^{\# \#} \mathrm{P}<0.01$ vs. I/R. 4 -PBA, 4-phenylbutyric acid; I/R, iscehemia/reperfusion; Cont, untreated control; CHOP, C/EBP homologous protein, Bcl-2, B cell lymphoma-2; BAX, Bcl-2-associated X protein; JNK, c-Jun N-terminal kinase; P-JNK, phosphorylated JNK.

apoptotic index between 5.6 and $16.7 \%(\mathrm{P}<0.01)$, compared with the control group. However, $5 \mathrm{mM} 4$-PBA pretreatment reduced this value to $7.5 \%$ ( $\mathrm{P}<0.01$, compared with I/R group; Fig. 3B). No significant differences were identified in apoptotic index between the 4-PBA-only treated group and the control group. As the change in $\Psi \mathrm{m}$ is one of the first signs of apoptosis, the present study determined the levels of mitochondrial depolarization using JC-1 staining (Fig. 3C). I/R significantly decreased the mitochondrial red:green fluorescence intensity ratio, indicating altered $\Psi_{\mathrm{m}}$ and depolarization. Treatment with 4-PBA partly recovered the mitochondrial red:green fluorescence intensity ratio, suggesting that it provided protection against the mitochondrial depolarization induced by I/R. In conclusion, these data confirmed that exposure of cardiomyocytes to I/R induced apoptotic cell death, however, pretreatment with 4-PBA reversed this effect and protected the cardiomyocytes.

ER stress-induced apoptosis was involved in the protective effect of 4-PBA on the myocardial I/R injury. To investigate whether the cardioprotective effect of 4-PBA is associated with the apoptosis induced by ER stress signaling during I/R injury, the protein levels of pro-apoptotic CHOP, BAX, JNK, and P-JNK, anti-apoptotic $\mathrm{Bcl}-2$, and the ER stress response proteins, Grp78, PERK and ATF6, were measured using Western blot analysis. CHOP is a critical pro-apoptotic factor in ER stress-associated apoptosis. As shown in Fig. 4A and B, the present study found that the myocardial protein expression of CHOP in the I/R group was significantly upregulated, compared with that in the control group $(\mathrm{P}<0.01)$. However, 4-PBA attenuated the I/R-induced upregulation in the expression of $\mathrm{CHOP}$, compared with the $\mathrm{I} / \mathrm{R}$ group $(\mathrm{P}<0.01)$. In addition, the $\mathrm{I} / \mathrm{R}$-induced increases in the expression levels of BAX (Fig. 4C) and P-JNK (Fig. 4D) were significantly inhibited by
4-PBA treatment. However, the inhibition of I/R on the protein expression of anti-apoptotic Bcl-2 (Fig. 4E) was attenuated by 4-PBA. To further understand the mechanism underlying the effect of 4-PBA on ER stress, the present study also examined the effect of 4-PBA on Grp78, PERK and ATF6, the major transcription factors involved in ER stress. The immunoblotting results indicated that the protein levels of Grp78, ATF6 and PERK were significantly increased following I/R treatment, which were attenuated by 4-PBA (Fig. 5). These data suggested that the ER stress response was activated acutely in I/R injury, whereas treatment with 4-PBA inhibited the activation of ER stress-associated proteins, compared with the respective control group. Taken together, these results demonstrated that the protective role of 4-PBA in I/R injury was associated with the inhibition of apoptosis signaling pathways induced by ER stress.

\section{Discussion}

In the present study, it was demonstrated that 4-PBA, an agent used clinically in disorders of the urea cycle in children and in sickle cell disease, exhibited cardioprotective effects during I/R through mediation of specific ER stress-induced cell apoptosis pathways. The involvement of apoptosis in cell loss, and the apoptotic signaling pathways in myocardial $\mathrm{I} / \mathrm{R}$ are relatively novel and controversial phenomena $(3,6,21)$, which remain to be fully elucidated. The present study examined whether I/R induced the UPR and whether induction of the UPR during I/R activates ER stress-associated cell death pathways. The resulting data demonstrated that I/R significantly increased apoptotic cardiomyocyte death by activating a series of pro-apoptotic proteins (Fig. 4). In addition, the expression levels of Grp78, ATF6 and PERK, which are important molecular 
A

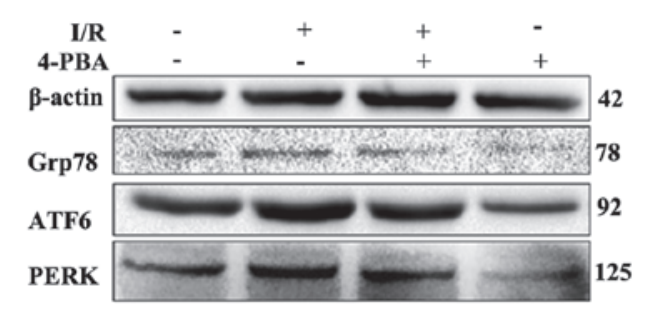

C

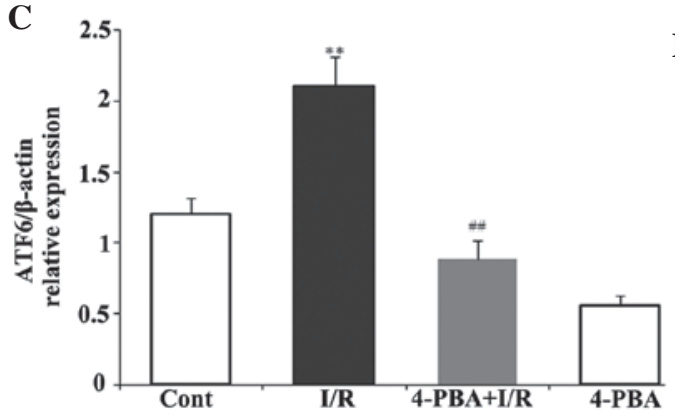

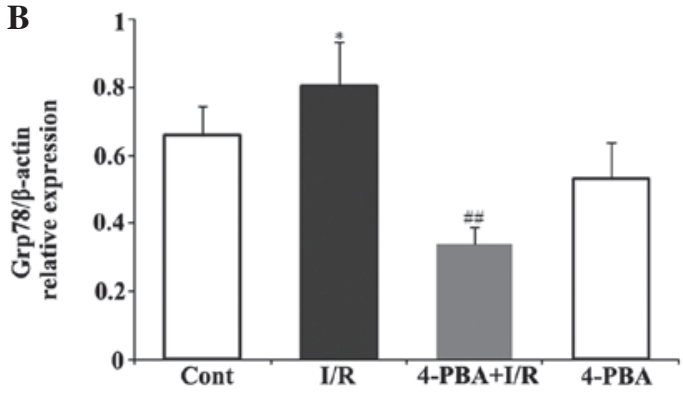

D

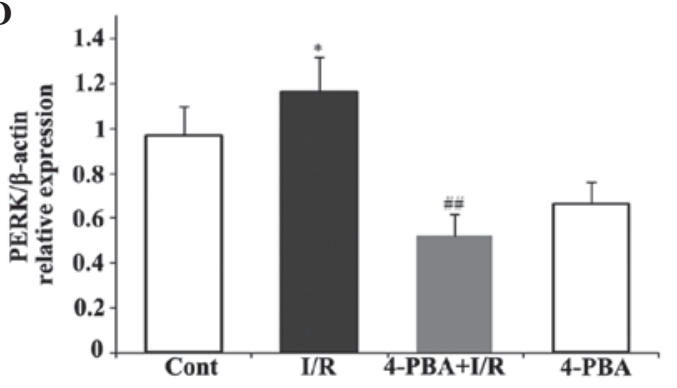

Figure 5. Involvement of ER stress signaling pathway in the cardioprotective effect of 4-PBA in I/R-induced H9c2 cells. The H9c2 cardiomyocytes were pretreated with or without 4-PBA $(5 \mathrm{mM})$ for $2 \mathrm{~h}$ prior to I/R exposure. (A) Expression levels of Grp78, ATF6 and PERK were detected by immunoblotting. The normalized ratio of (B) Grp78, (C) ATF6 and (D) PERK to $\beta$-actin, were determined by densitometry. The results are presented as the mean \pm standard deviation from three independent experiments. ${ }^{*} \mathrm{P}<0.05$ and ${ }^{* *} \mathrm{P}<0.01$, vs. Cont; ${ }^{\# \#} \mathrm{P}<0.01$, vs. I/R. 4-PBA, 4-phenylbutyric acid; I/R, iscehemia/reperfusion; Cont, untreated control; Grp78, glucose-regulated protein 78; ATF6, activating transcription factor 6; PERK, protein kinase RNA-like endoplasmic reticulum kinase.

indicators of ER stress, were significantly upregulated in the I/R group (Fig. 5), indicating that ER stress was induced by I/R. However, the overexpression of the ER stress molecules was significantly suppressed by 4 -PBA treatment at an appropriate dose.

4-PBA is a chemical chaperone with approved in vivo safety and has already been approved by the US Food and Drug Administration for clinical use (22). Numerous studies have demonstrated that 4-PBA inhibits ER stress and UPR signaling. A previous study showed that 4-PBA significantly downregulates the expression levels of fibrosis-associated genes and protects against cardiac fibrosis in an animal model to withstand pressure overload (23). According to another report, the attenuation of ER stress using 4-PBA prevents oproterenol-induced cardiac fibrosis (24). The aim of the present study was to confirm the hypothesis that 4-PBA protects cardiomyocytes from I/R injury by inhibiting the accumulated misfolded protein-induced ER stress response and the associated apoptosis.

The ER, as a vast membranous organelle, is responsible for the homeostasis of cellular $\mathrm{Ca}^{2+}$, and the synthesis, maturation and trafficking of a wide range of proteins (25). The capacity of the ER is important for the cell due to its capabilities in managing synthetic, metabolic and other adverse conditions (26). Recognition of the contribution of ER to apoptotic cell death has been developed. It was reported in 2000 for the first time that treatment with calcium ionophores, a sarcoplasmic/ER-calcium ATPase pump inhibitor (thapsigargin), or an inhibitor of N-linked glycosylation (tunicamycin) can initiate a form of apoptosis referred to as ER stress-mediated apoptosis (27). In the ER stress signaling cascade, the chaperone, Grp78, is the first element, which is involved in protein folding and, consequently, is responsible for activating the three PERK, ATF6 and IRE1 UPR sensors $(8,28)$. Increased numbers of unfolded proteins within the ER prompt Grp78 to 'undock' from IRE1, PERK and ATF6. ATF6 undergoes proteolytic activation in the Golgi apparatus, followed by nuclear translocation, where it dimerizes with basic leucine zipper transcription factors, including X-box binding protein 1, which regulate ER stress response genes (29). PERK oligomerization activates its intrinsic kinase activity, resulting in eukaryotic translation initiation factor 2 , subunit $1 \alpha$ (eIF $2 \alpha$ ) phosphorylation, which suppresses translation. The phosphorylation of eIF $2 \alpha$ promotes pro-survival (early) and pro-apoptotic (late) transcriptional programs (30). Several studies have documented activation of the UPR in the myocardium (31). A common element following activation of these three sensors is the expression of the protein CHOP, which has been documented to mediate apoptosis following ER stress and to be involved in several ER-stress-associated diseases $(12,32)$. In the present study, it was demonstrated that the expression levels of Grp78, ATF6 and PERK were significantly elevated in the I/R group. Of note, it was found that 4-PBA effectively inhibited ER stress-mediated apoptosis pathways. 4-PBA treatment not only mediated the protein expression levels of BAX and Bcl-2 in vitro, but it also reduced the expression levels of ER stress moderators, Grp78, ATF6 and PERK, and CHOP in particular. $\mathrm{I} / \mathrm{R}$ induces a prominent increase in $\mathrm{CHOP}$ activation, a common effect of the three main signaling pathways of ER stress, in short time, which triggers JNK- regulated apoptosis. The phosphorylation of PERK in response to ER stress is considered to be a protective response, as it attenuates protein synthesis and provides cells with more time to manage correct folding or degrade accumulated misfolded proteins (33). Thus, the lack of PERK- kinase activity and lack of eIF $2 \alpha$ 
phosphorylation make the cells more vulnerable to apoptosis in response to ER stress. The ATF6 pathway is considered to control a rapid response to stimulus-induced ER stress (25). Western blot analysis identified 92 and $125 \mathrm{kDa}$ processed fragments, confirming the activation of ATF6 and PERK in I/R cardiomyocytes (Fig. 5C and D). Members of the Bcl-2 family are also important in the transduction of an internal apoptotic signal.

In conclusion, the present study reported for the first time, to the best of our knowledge, that I/R induced the ER stress-associated pathways and their downstream apoptotic targets. The mechanism by which I/R affects the homeostasis of ER and induces ER stress remains to be fully elucidated, and only indirect evidence is available to suggest that $I / R$ in the heart induces ER stress. In the present study, the results showed the inhibitory effect of 4-PBA on decreasing myocardium apoptosis and preventing cell death induced by $\mathrm{I} / \mathrm{R}$, suggesting the possibility of the use of 4-PBA as a therapeutic agent for cardiac ischemia/reperfusion diseases. However, the mechanism underlying the cardioprotective action of 4-PBA remains to be fully elucidated. Further investigations are required to elucidate other mechanisms involved in the effect of 4-PBA in ER stress, and the distinct effects of cardiac injury and heart failure. Improved understanding may lead to the design of improved ER stress-targeted therapies against life-threatening cardiac diseases.

\section{References}

1. Di Diego JM and Antzelevitch C: Acute myocardial ischemia: Cellular mechanisms underlying ST segment elevation. J Electrocardiol 47: 486-490, 2014.

2. Logue SE, Gustafsson AB, Samali A and Gottlieb RA Ischemia/reperfusion injury at the intersection with cell death. J Mol Cell Cardiol 38: 21-33, 2005.

3. Minamino $\mathrm{T}$ and Kitakaze M: ER stress in cardiovascular disease. J Mol Cell Cardiol 48: 1105-1110, 2010.

4. Scarabelli TM and Gottlieb RA: Functional and clinical repercussions of myocyte apoptosis in the multifaceted damage by ischemia/reperfusion injury: Old and new concepts after 10 years of contributions. Cell Death Differ 11 (Suppl 2): S144-S152, 2004.

5. Jennings RB, Sommers HM, Smyth GA, Flack HA and Linn H: Myocardial necrosis induced by temporary occlusion of a coronary artery in the dog. Arch Pathol 70: 68-78, 1960.

6. Duan SR, Wang JX, Wang J, Xu R, Zhao JK and Wang DS: Ischemia induces endoplasmic reticulum stress and cell apoptosis in human brain. Neurosci Lett 475: 132-135, 2010.

7. Kim I, Xu W and Reed JC: Cell death and endoplasmic reticulum stress: Disease relevance and therapeutic opportunities. Nat Rev Drug Discov 7: 1013-1030, 2008.

8. Shen X, Zhang K and Kaufman RJ: The unfolded protein response-a stress signaling pathway of the endoplasmic reticulum. J Chem Neuroanat 28: 79-92, 2004.

9. Ron D and Walter P: Signal integration in the endoplasmic reticulum unfolded protein response. Nat Rev Mol Cell Biol 8 519-529, 2007.

10. Nishitoh H: Life and death under the ER stress condition. Journal of Oral Biosciences 46: 259-269, 2004.

11. Sozen E, Karademir B and Ozer NK: Basic mechanisms in endoplasmic reticulum stress and relation to cardiovascular diseases. Free Radic Biol Med 78: 30-41, 2015.

12. Verfaillie T, Garg AD and Agostinis P: Targeting ER stress induced apoptosis and inflammation in cancer. Cancer Lett 332: 249-264, 2013

13. Brusilow SW and Maestri NE: Urea cycle disorders: Diagnosis, pathophysiology and therapy. Adv Pediatr 43: 127-170, 1996.

14. Qi X, Hosoi T, Okuma Y, Kaneko M and Nomura Y: Sodium 4-phenylbutyrate protects against cerebral ischemic injury. Mol Pharmacol 66: 899-908, 2004
15. Vilatoba M, Eckstein C, Bilbao G, Smyth CA, Jenkins S, Thompson JA, Eckhoff DE and Contreras JL: Sodium 4-phenylbutyrate protects against liver ischemia reperfusion injury by inhibition of endoplasmic reticulum-stress mediated apoptosis. Surgery 138: 342-351, 2005.

16. Wu CX, Liu R, Gao M, Zhao G, Wu S, Wu CF and Du GH: Pinocembrin protects brain against ischemia/reperfusion injury by attenuating endoplasmic reticulum stress induced apoptosis. Neurosci Lett 546: 57-62, 2013.

17. Mimori S, Ohtaka H, Koshikawa Y, Kawada K, Kaneko M, Okuma Y, Nomura Y, Murakami Y and Hamana $\mathrm{H}$ : 4-Phenylbutyric acid protects against neuronal cell death by primarily acting as a chemical chaperone rather than histone deacetylase inhibitor. Bioorg Med Chem Lett 23: 6015-6018, 2013.

18. Kuang X, Hu W, Yan M and Wong PK: Phenylbutyric acid suppresses protein accumulation-mediated ER stress in retrovirus-infected astrocytes and delays onset of paralysis in infected mice. Neurochem Int 57: 738-748, 2010.

19. Sun J, Sun G, Meng X, Wang H, Wang M, Qin M, Ma B, Luo Y, Yu Y, Chen R, et al: Ginsenoside RK3 prevents hypoxia-reoxygenation induced apoptosis in $\mathrm{H} 9 \mathrm{c} 2$ cardiomyocytes via AKT and MAPK pathway. Evid Based Complement Alternat Med 2013: 690190, 2013.

20. Sun L, Isaak CK, Zhou Y, Petkau JC, O K, Liu Y and Siow YL: Salidroside and tyrosol from Rhodiola protect H9c2 cells from ischemia/reperfusion-induced apoptosis. Life Sci 91: 151-158, 2012

21. Yuan Y, Guo Q, Ye Z,Pingping X, Wang N and Song Z: Ischemic postconditioning protects brain from ischemia/reperfusion injury by attenuating endoplasmic reticulum stress-induced apoptosis through PI3K-Akt pathway. Brain Res 1367: 85-93, 2011.

22. Kim HD, Jang CY, Choe JM, Sohn J and Kim J: Phenylbutyric acid induces the cellular senescence through an Akt/p21(WAF1) signaling pathway. Biochem Biophys Res Commun 422: 213-218, 2012.

23. Park CS, Cha H, Kwon EJ, Sreenivasaiah PK and Kim do H: The chemical chaperone 4-phenylbutyric acid attenuates pressure-overload cardiac hypertrophy by alleviating endoplasmic reticulum stress. Biochem Biophys Res Commun 421: 578-584, 2012.

24. Ayala P, Montenegro J, Vivar R, Letelier A, Urroz PA, Copaja M, Pivet D, Humeres C, Troncoso R, Vicencio JM, et al: Attenuation of endoplasmic reticulum stress using the chemical chaperone 4-phenylbutyric acid prevents cardiac fibrosis induced by isoproterenol. Exp Mol Pathol 92: 97-104, 2012.

25. Yamamoto K, Yoshida H, Kokame K, Kaufman RJ and Mori K: Differential contributions of ATF6 and XBP1 to the activation of endoplasmic reticulum stress-responsive cis-acting elements ERSE, UPRE and ERSE-II. J Biochem 136: 343-350, 2004.

26. Wang WA, Groenendyk J and Michalak M: Endoplasmic reticulum stress associated responses in cancer. Biochim Biophys Acta 1843: 2143-2149, 2014.

27. Powell KS and Latterich M: The making and breaking of the endoplasmic reticulum. Traffic 1: 689-694, 2000.

28. Cullinan SB and Diehl JA: Coordination of ER and oxidative stress signaling: The PERK/Nrf2 signaling pathway. Int J Biochem Cell Biol 38: 317-332, 2006.

29. Ye J, Rawson RB, Komuro R, Chen X, Davé UP, Prywes R, Brown MS and Goldstein JL: ER stress induces cleavage of membrane-bound ATF6 by the same proteases that process SREBPs. Mol Cell 6: 1355-1364, 2000.

30. Owen CR, Kumar R, Zhang P, McGrath BC, Cavener DR and Krause GS: PERK is responsible for the increased phosphorylation of eIF2alpha and the severe inhibition of protein synthesis after transient global brain ischemia. J Neurochem 94: 1235-1242, 2005.

31. Zhu S, Jin J, Wang Y, Ouyang Z, Xi C, Li J, Qiu Y, Wan J, Huang $M$ and Huang $Z$ : The endoplasmic reticulum stress response is involved in apoptosis induced by aloe-emodin in HK-2 cells. Food Chem Toxicol 50: 1149-1158, 2012.

32. Li H, Zhu X, Fang F, Jiang D and Tang L: Down-regulation of GRP78 enhances apoptosis via CHOP pathway in retinal ischemia-reperfusion injury. Neurosci Lett 575: 68-73, 2014.

33. Vaughn LS, Snee B and Patel RC: Inhibition of PKR protects against tunicamycin-induced apoptosis in neuroblastoma cells. Gene 536: 90-96, 2014. 